\title{
CS Research Square \\ SPOP Could Play a Potential Inhibitory Role in Human Renal Cell Carcinoma
}

\section{zhi chen}

People's Hospital of Guangxi Zhuang Autonomous Region

\section{Zuan Li}

People's Hospital of Guangxi Zhuang Autonomous Region

\section{Deyong Nong}

People's Hospital of Guangxi Zhuang Autonomous Region

\section{Ximing Li}

People's Hospital of Guangxi Zhuang Autonomous Region

\section{Guihai Huang}

People's Hospital of Guangxi Zhuang Autonomous Region

Junhao Lin

People's Hospital of Guangxi Zhuang Autonomous Region

Wei Li (D liwei95_2000@163.com )

People's Hospital of Guangxi Zhuang Autonomous Region

\section{Research}

Keywords: Renal cell carcinoma, SPOP, In vitro proliferation, migration, invasion, apoptosis

Posted Date: May 7th, 2021

DOl: https://doi.org/10.21203/rs.3.rs-395336/v1

License: (1) (1) This work is licensed under a Creative Commons Attribution 4.0 International License.

Read Full License 


\section{Abstract}

Background: SPOP, a substrate adaptor of Cul3 ubiquitin ligase, plays crucial roles in solid neoplasms by promoting the ubiquitination and degradation of substrates. Limited studies have shown that SPOP is overexpressed in human renal cell carcinoma (RCC) tissue. However, the exact role of SPOP in RCC remains unclear and needs to be further elucidated. The present study showed that SPOP was expressed at different levels in different RCC cell lines. The purpose of this study was to explore the roles of SPOP in the biological features of RCC cells and determine the expression levels of SPOP in human tissue microarrays (TMAs) and kidney tissues.

Methods: Here, SPOP was overexpressed by lentiviral vector transfection in ACHN and Caki-1 cells, and SPOP was knocked down in Caki-2 cells with similar transfection methods. The transfection efficiency was evaluated by quantitative PCR and western blotting analyses. The role of SPOP in the proliferation, migration, invasion and apoptosis of cell lines was determined by the MTT, wound-healing, Transwell and flow cytometry assays. Moreover, the cells were treated with different drug concentrations in proliferation and apoptosis assays to investigate the effect of sunitinib and IFN-a2b on the proliferation and apoptosis of SPOP-overexpressing cells and SPOP-knockdown RCC cells. Finally, immunohistochemical staining of SPOP was performed in kidney tissues and TMAs, which included RCC tissues and corresponding adjacent normal tissues.

Results: Overexpression of SPOP inhibited cell proliferation, migration and invasion and increased cell apoptosis. Interestingly, sunitinib and IFN- $a 2 b$ at several concentrations increased the proliferation inhibitory rate and total apoptosis rate of cells overexpressing SPOP. The findings of the present study showed that the SPOP protein was significantly expressed at low levels in most clear cell RCC (CCRCC) tissues and at relatively high levels in the majority of adjacent normal tissues and kidney tissues. KaplanMeier survival analysis showed that there was no statistically significant difference in cumulative survival based on the data of different SPOP expression levels in TMA and patients.

Conclusions: In contrast to previous studies, our findings demonstrated that overexpression of SPOP might suppress the progression of RCC cells, which was supported by cell experiments and immunohistochemical staining. SPOP could be a potential tumour inhibitor in RCC.

\section{Background}

Renal cell carcinoma (RCC) ranks as the sixth most frequently diagnosed cancer in men and the 10th most frequently diagnosed cancer in women in the United States. Moreover, it has been reported that RCC incidence and mortality are increasing around the world[1-3]. Clear cell carcinoma represents the most common tissue subtype, accounting for $70 \%$ $90 \%$ of RCCs. Approximately $25 \%$ of patients diagnosed with RCC have evidence of advanced disease or metastases, although the increasing incidence is correlated with the wide use of multiple medical techniques for RCC screening[4, 5]. Primary localized renal cancers can be cured by radical nephrectomy. The treatment outcome for patients with metastatic 
RCC (mRCC) has improved since the introduction of VEGF inhibitors and agents targeting the $\mathrm{PI} 3 \mathrm{~K} / \mathrm{Akt} / \mathrm{mTOR}$ signalling pathway, including combination strategies, such as lenvatinib and everolimus; however, drug resistance is still a major problem. Unfortunately, previous systemic treatment options could not provide long-term efficacy for mRCC, which ultimately becomes resistant to first-line drugs[68]. The molecular mechanism of resistance to targeted therapy for advanced or mRCC has become a research hotspot.

Speckle-type POZ protein (SPOP), a novel nuclear speckle-type protein, was first identified in 1997[9]. Previous studies have shown that SPOP, as a substrate adaptor of cullin3-RING ubiquitin ligase (CRL3), recruits substrates to CRL3 for ubiquitination and degradation, such as the androgen receptor (AR), steroid receptor coactivator (SRC)-3 and PTEN proteins[10-12]. Over the past decades, SPOP has been confirmed as a tumour suppressor in several cancers, including prostate cancer, lung cancer and gastric cancer, and studies of the differential expression levels and mutation status of SPOP have indicated that SPOP plays different roles in cancer cell development[13]. It is well known that dramatically decreased SPOP expression is negatively correlated with tumorigenesis in gastric cancer tissues[14]. In addition, SPOP gene mutation is the most common missense point mutation in prostate cancer and affects the progression of prostate tumours through coordinated regulation of the PI3K/mTOR and AR signalling pathways[15, 16]. Interestingly, to date, mutation of SPOP has not been found in RCC tumours[11, 16]. However, some studies have shown overexpression of SPOP in the cytoplasm of clear cell RCC (cCRCC) cells and have indicated some correlation with high pathological stages, lymph node invasion and metastasis[17, 18]. However, the exact role of SPOP in the biological features of RCC and its potential molecular mechanism in RCC tumours remain unclear. Based on the results of preliminary cell experiments, SPOP might act as a protective factor in RCC.

\section{Materials And Methods}

\section{Cell lines and cell culture}

ACHN and Caki-1 cell lines were obtained from the Cell Bank of Type Culture Collection of Chinese Academy of Sciences. Caki-2 cell lines were provided by Guangzhou Cell cook Biotech Co. Ltd. ACHN, Caki-1 and Caki-2 cell lines were maintained in Dulbecco's modified Eagle's medium (DMEM) (Gibco) supplemented with $10 \%$ foetal bovine serum (FBS). All the cells were cultured in a humidified incubator containing $5 \% \mathrm{CO}_{2}$ at $37^{\circ} \mathrm{C}$ and were used in further experiments.

\section{Transfections}

The plasmids were transfected into cells with Lipofectamine 2000 according to the manufacturer's instructions. The following day, the cells were cultured with media containing neomycin and selected for two weeks to obtain stably transfected cells. The SPOP plasmid or shRNA plasmid was packaged and transfected into retroviral packaging cells. Retroviral supernatants were added to the cells, spun for 45 min at $1800 \mathrm{rpm}$ and incubated for $4 \mathrm{~h}$ at $37^{\circ} \mathrm{C}$. The cell medium was switched to medium 
supplemented with puromycin for one week to select stable cell lines. The overexpression and knockdown efficiency of SPOP in the cells was tested by western blot and QPCR analyses.

\section{Western blot analysis}

As previously described[19], cell protein samples were harvested using RIPA buffer, and $20 \mu \mathrm{g}$ of the protein sample was separated on $12 \%$ SDS-polyacrylamide gels followed by wet transfer at room temperature. The blots were then blocked with non-fat milk, followed by incubation with diluted primary antibody overnight at $4^{\circ} \mathrm{C}$. The membranes were washed three times with TBST for 5 min and subsequently incubated with the appropriate secondary antibody conjugated to IRDye800 at room temperature for $2 \mathrm{~h}$. Protein bands were visualized with the ECL detection system and analysed using ImageJ software.

\section{Quantitative real-time PCR analysis}

Total RNA from cells was extracted and reverse transcribed using a cDNA synthesis kit (Invitrogen). Realtime PCR analysis was performed using a LightCycler 96 (Roche). The peak of the melting curve was defined as the criterion for amplification specificity. The relative expression levels of mRNAs were determined by normalization to the expression levels of the internal control gene GAPDH, and the data were analysed by the $\Delta \Delta \mathrm{Ct}$ method.

\section{Cell invasion assay}

The capacity of cell invasion was evaluated by the Transwell assay. A total of $2 \times 10^{5}$ cells in serum-free medium were plated on top of the Transwell chamber, which was coated with Matrigel matrix (Corning 354230). Medium supplemented with $10 \%$ FBS as the chemoattractant was added to the bottom of the chamber. The cells were then incubated in Transwell plates at $37^{\circ} \mathrm{C}$ in $5 \% \mathrm{CO}_{2}$ for $48 \mathrm{~h}$. The non-invading cells at the top of the chamber were carefully removed with a cotton swab. The cells on the lower surface of the Transwell chamber were stained with crystal violet for $30 \mathrm{~min}$ after fixation with paraformaldehyde. The inserts were washed three times with PBS, and the number of invading cells was counted under a microscope.

\section{Cell migration assay}

Cell migration was determined by the wound-healing assay. A total of $1.2 \times 10^{5}$ cells were plated in a 12 well plate at $37^{\circ} \mathrm{C}$ and $5 \% \mathrm{CO}_{2}$ overnight. A horizontal scratch was then made in the plate using a sterile pipette tip, followed by washing with PBS three times to remove the floating cells. Finally, the cells were incubated in serum-containing medium at $37^{\circ} \mathrm{C}$ in $5 \% \mathrm{CO}_{2}$ for $24 \mathrm{~h}$. The scratch migration area was calculated using Image J software after $0 \mathrm{~h}$ and $24 \mathrm{~h}$.

\section{Cell proliferation and apoptosis assays}

For the proliferation assay, $1.5 \times 10^{5}$ cells were cultured in 96 -well plates with regular medium at $37^{\circ} \mathrm{C}$ and $5 \% \mathrm{CO}_{2}$ for $24 \mathrm{~h}$. The next day, the culture medium was replaced with medium supplemented with IFNa2b $(20,80,4000$ and $5000 \mathrm{IU} / \mathrm{ml})$ or sunitinib $(2.50,5.01,7.00$ and $10.05 \mu \mathrm{mol} / \mathrm{L})$ and incubated for 48 
h. During culture, $10 \mathrm{ml}$ CCK-8 chromogenic agent and $100 \mathrm{ml}$ DMEM without FBS were added to the wells and incubated for $1 \mathrm{~h}$. The absorbance $(A)$ at $450 \mathrm{~nm}$ was analysed using a microplate reader. The inhibitory rate of cell growth (\%) was quantified as follows: $\left(1-\left(A_{\text {treated }}\right) /\left(A_{\text {control }}\right)\right) \times 100 \%$. For the apoptosis assay, $1 \times 10^{5}$ cells were seeded into 6 -well plates, allowed to attach overnight and were treated with $10 \%$ FBS (control), IFN-a2b (20, 40 and $80 \mathrm{IU} / \mathrm{ml})$, or sunitinib $(5.0,5.5$ and $6.0 \mu \mathrm{mol} / \mathrm{L})$ for $48 \mathrm{~h}$. The cells were collected by centrifugation at $1200 \mathrm{rpm}$ for $5 \mathrm{~min}$ at $37^{\circ} \mathrm{C}$. The collected cells were washed with PBS and $50 \mu \mathrm{l} 1 \mathrm{x}$ binding buffer. Then, the cells were stained with Annexin V-APC and 7-AAD and incubated at room temperature in the dark for $15 \mathrm{~min}$. Subsequently, $50 \mu \mathrm{l} 1 \mathrm{x}$ binding buffer was added, and the samples were tested using an Accuri ${ }^{\mathrm{TM}} \mathrm{C} 6$ PLUS flow cytometer (BD Biosciences). The sum of early apoptosis and late apoptosis was defined as total apoptosis.

\section{Tissue microarrays (TMA) and normal kidney tissues}

TMAs of formalin-fixed paraffin-embedded human renal tumour tissue and adjacent normal tissue from the Shanghai Outdo Biotech Company were evaluated. The TMA comprised 180 tissue samples (90 tumour tissue samples and 90 adjacent normal tissue samples) collected from patients who underwent nephrectomy between 2006 and 2008. The 90 RCC specimens included clear cell renal cells $(n=88)$ and papillary cells $(n=2)$. In addition, normal kidney tissues $(n=10)$ obtained from The People's Hospital of Guangxi Zhuang Autonomous Region were used. Related clinical data, including follow-up time, sex, and tumour stage, were recorded in detail for all patients who signed the informed consent form. Moreover, the above tissue samples used in this experiment were obtained upon ethical approval.

\section{Immunohistochemical staining}

Immunohistochemical staining of the SPOP protein in the cytoplasm of TMA tissues and kidney tissues was performed with appropriate antibodies according to the methods of a previous study[20]. Briefly, paraffin-embedded sections were subjected to deparaffinization, rehydration, and heat-induced antigen retrieval. The sections were incubated with primary SPOP antibody overnight at $4^{\circ} \mathrm{C}$ after blocking endogenous peroxidase activity with $3 \%$ hydrogen peroxide. Rabbit IgG antibody was used for the isotype control. 3,3'-Diaminobenzidine (DAB) was added as a chromogen followed by counterstaining with haematoxylin. The staining intensity and positive staining rate were assessed by two independent pathologists according to the histologic scoring system (H-score). SPOP expression was scored comprehensively based on the positive staining rate and staining intensity. The positive staining rate was scored as follows: 0 (negative), $1+(1-25 \%), 2+(26-50 \%), 3+(51-75 \%)$, and $4+(76-100 \%)$. The intensity of cytoplasmic staining was classified as follows: 0 (negative), 1+ (weak), 2+ (moderate), and 3+ (strong). The above two scores were multiplied to obtain the final score. A total score of SPOP immunohistochemical staining $\geq 6$ was defined as high expression; otherwise, it was considered as low expression.

\section{Statistical analysis}

Statistical analyses and figure preparation were performed using SPSS 24.0 (SPSS, Inc, Chicago, IL, USA) and GraphPad Prism 7.0 (San Diego, California, USA) software. Values of the in vitro cell experiments are 
presented as the mean \pm standard deviation based on results obtained from at least three independent experiments. Comparisons were made between homogeneous experimental groups using the t-test or ANOVA, as appropriate. Mann-Whitney $U$ test were used to analyse the differences of SPOP expression between tumor tissues and adjacent normal tissues. Survival analysis was determined by the KaplanMeier method and compared by the log rank test. A p-value less than 0.05 was considered to be statistically significant.

\section{Results}

\section{Overexpression of SPOP inhibits the invasion and migration of RCC cells in vitro}

ACHN, Caki-1 and Caki-2 cell lines are commonly used in RCC studies. Our preliminary western blotting results showed that SPOP expression was significantly downregulated in ACHN and Caki-1 cell lines and upregulated in the Caki-2 cell line (Fig. 1A and B). To explore the effect of SPOP on the biological features of RCC cells, ACHN and Caki-1 cells were transfected using a lentiviral vector overexpressing SPOP, and SPOP in Caki-2 cells was knocked down with similar transfections using a small hairpin RNA (shRNA) lentiviral vector. The overexpression and knockdown efficiency of SPOP were verified by western blot (Fig. 1C and D) and QPCR analyses. By Transwell assays, we found that overexpression of SPOP in ACHN and Caki-1 cells significantly inhibited the invasive ability of the cells after incubation for $48 \mathrm{~h}$ compared with the negative control (NC). Similar results were observed in the si-NC group of Caki-2 cells compared with the SPOP-silenced group $(\mathrm{P}<0.0001$, Fig. $2 \mathrm{~A})$. Moreover, cell migration is another malignant behaviours of cancer cells. The effect of SPOP on cell migration was measured by the woundhealing assay. High expression of SPOP in Caki-1 and Caki-2 cells significantly decreased the migration capacity of RCC cells after $24 \mathrm{~h}$ compared to that of cells with low expression of SPOP $(P<0.01$ Fig. $2 B)$. A similar phenomenon was observed in ACHN cells; however, no significant difference was noted $(P=$ 0.0649). The above findings suggested that SPOP may play an important role in suppressing the malignant biological behaviour of RCC cells.

\section{SPOP suppresses RCC cell proliferation and induces cellular apoptosis}

The data showed that SPOP plays a key role in the invasion and migration of RCC cells. Next, we performed experiments to investigate the effect of SPOP on the proliferation and apoptosis of RCC cells by using the MTT assay and flow cytometry. Previous studies suggested that the motility of Caki-2 cells was stronger than that of ACHN and Caki-1 cells, which could be suppressed by IFN or sorafenib, especially when the two drugs were combined, which indicated that Caki-2 cells were more aggressive than ACHN and Caki-1 cells[21]. Advanced or mRCC cannot be cured due to drug resistance, which remains one of the most challenging issues. In the present study, we investigated the effects of first-line drugs (sunitinib and IFN- $a 2 b$ ) on the above cell lines with different SPOP expression levels. The results showed that overexpression of SPOP decreased RCC cell proliferation (Fig. 3A and B) and induced 
cellular apoptosis under several drug concentrations (Fig. 3C and D) compared to low SPOP expression. All of these findings suggested that the SPOP protein may improve the susceptibility of RCC cells to drug treatments.

\section{SPOP is mainly expressed at low levels in the cytoplasm of CcRCC tissue}

Cell experiments indicated that SPOP expression significantly inhibited the growth and progression of RCC cells. To determine SPOP expression in human RCC tissue and normal kidney samples, immunohistochemical staining was performed on TMA tissues consisting of ccRCC tissues $(n=88)$, papillary RCC tissues $(n=2)$ and corresponding adjacent normal tissues. We found that the expression of SPOP protein in the cytoplasm was significantly downregulated in $83 \%$ of ccRCC tumour tissues and upregulated in $88 \%$ of adjacent nontumour tissues $(P<0.0001$,Fig. 4$)$. To further confirm these preliminary results, we continued to analyse SPOP expression in the normal kidney samples. Consistent findings were observed in the immunohistochemical staining of normal kidney tissues (Fig. 4), in which the SPOP protein was mainly overexpressed in the cytoplasm of kidney tissues. This is quite different from previous studies, which showed that the SPOP protein was overexpressed in over $80 \%$ of RCC tissues, even in nearly $100 \%$ of primary ccRCCs showing SPOP accumulation, and negative in $82 \%$ of normal kidney tissues[17, 18, 22].

\section{SPOP protein expression and clinical correlations in RCC}

Based on the immunohistochemical results, we showed that the SPOP protein was expressed at lower levels in most RCC tissues. Next, we investigated whether SPOP expression was associated with overall survival. Survival analysis was performed using the Kaplan-Meier method based on SPOP expression levels and follow-up time. We found that there was no significant correlation between SPOP expression and patient cumulative survival $(P>0.05)$, as shown in Fig. 5. Analysis of Kaplan-Meier curves suggested that the role of SPOP in RCC remains controversial, and the mechanism of action of SPOP needs to be explored further. However, all of our findings suggest that the SPOP protein could act as a protective factor in RCC.

\section{Discussion}

SPOP, a CRL3 substrate adaptor protein, plays an important role in the development of some cancers[1012]. Over the past decade, the potential functions of SPOP in urologic cancers have gradually attracted much attention from investigators. SPOP was found to be the most common missense mutated gene in human prostate cancers and has been shown to be associated with the pathogenesis of primary prostate tumours, but SPOP mutations in RCC tumours have not yet been reported[15, 16, 23, 24]. Recent studies have shown that SPOP is an oncoprotein that is overexpressed in RCC $17,18,25]$. However, the results of our preliminary cell experiments showed that overexpression of SPOP inhibited RCC cell proliferation, migration and invasion and increased cellular apoptosis rates. Similar to our cell experiment results, some studies also found that high expression of SPOP suppressed the malignant biological behaviour of 
cancer cells in vitro via ubiquitin-dependent proteolysis of the signalling pathway[14, 26-28]. Therefore, SPOP may be associated with inhibition of the aggressiveness of RCC cells.

SPOP plays key roles in cancer development by promoting ubiquitination and degradation of the substrate protein of specific signalling pathways. For example, SPOP has a definitive tumour suppressing role in gastric cancer by promoting the degradation of the transcription factor Gli2 of the Hedgehog $(\mathrm{Hh}) / \mathrm{Gli2}$ signalling pathway[14]. In an in vitro drug sensitivity experiment, we found that the proliferation inhibitory rates of cells were significantly increased and cellular apoptosis was induced when SPOP was overexpressed in RCC cells that were treated with sunitinib or IFN-a2b. Sunitinib, a tyrosine kinase inhibitor targeting the VEGF receptor, has been the first-line targeted therapy for patients with mRCC who have been classified as having MSKCC intermediate-risk or poor-risk disease and has shown an improvement in survival[6,29]. Cellular migration, proliferation and survival of cancer cells as well as endothelial cell differentiation are driven mainly by VEGF/VEGFR activation, which in turn activates the $\mathrm{PI} 3 \mathrm{~K} / \mathrm{Akt} / \mathrm{mTOR}$ signalling pathway[30, 31]. One study showed that 4-chloro fascaplysin, a marine sponge alkaloid derivative, inhibited tumour growth and VEGF-mediated angiogenesis by disrupting the $\mathrm{PI3K} / \mathrm{Akt} / \mathrm{mTOR}$ signalling cascade[32]. The PI3K/Akt/mTOR axis, which is involved in cancer cell proliferation, differentiation and cellular metabolism, is frequently activated in many cancers and is one of the most significant molecular pathways in $\mathrm{mRCC}[33,34]$. Activation of the PI3K/Akt/mTOR pathway is correlated with aggressive behaviour and poor prognosis of RCC tumours and is more significantly altered in CCRCC, high TNM stage tumours, and tumours with poor prognostic features[35, 36]. SPOP binding to the substrate is a crucial event for E3 ligase-mediated ubiquitination and subsequent proteasome degradation. Levels of the PI3K/Akt pathway have been found to be correlated with SPOP expression, which could inhibit colorectal cancer and osteosarcoma invasion by significantly reducing the levels of PI3K and p-Akt[37, 38]. In the present study, there was a significant difference in the sensitivity of different cell lines overexpressing SPOP to several concentrations of sunitinib. It could be a promising potential molecular mechanism that may provide an effective therapeutic strategy for patients with advanced kidney cancer by exploring the relationships among the SPOP, VEGF and PI3K/Akt/mTOR pathways.

In addition, the Hedgehog signalling pathway, which increases tumour invasion and metastatic potential, is another important molecular mechanism that is worth investigating in the future. Aberrant activation of the Hedgehog pathway is associated with tumorigenesis in some cancers, including RCC, and plays an important role in RCC development[39-41]. Limited studies suggest that SPOP suppresses tumour development by negatively regulating the Hedgehog/Gli2 signalling pathway in gastric cancer[14]. In addition, the expression levels of the Hedgehog signalling pathway component genes Gli1 and Gli2, which are activated by the PI3K/Akt signalling pathway in RCC, are significantly elevated in CCRCC and provide a promising therapeutic strategy for RCC[42]. Currently, the Hedgehog inhibitors (HHIs) vismodegib and sonidegib are approved for use in advanced BCC, and other potential uses for the treatment of solid tumours beyond BCC are under development or in clinical trials[43]. Given the above, SPOP, as a tumour suppressor protein, plays an important role in inhibiting tumorigenesis by regulating different signalling pathways. However, studies on the molecular mechanism of the SPOP protein in RCC 
are still limited. Exploring the underlying mechanisms of signalling pathways in kidney cancer in detail is the best approach to provide a theoretical basis for the development of novel therapeutic strategies for mRCC patients in the future.

Differential expression levels or mutation profiles of SPOP in tumours play different roles in tumorigenesis and cancer progression $[10,11,13]$. Several studies have shown that SPOP expression is downregulated in some primary tumours, including gastric cancer, liver cancer, colorectal cancer, pancreatic cancer and non-small cell lung cancer, and low expression of SPOP is associated with poor prognosis in patients[14, 26-28, 44]. In the current study, immunohistochemical staining demonstrated that the SPOP protein was mainly expressed at low levels in the cytoplasm of ccRCC tissues and was relatively highly expressed in most adjacent nontumour tissues and normal kidney tissues. The inhibitory role of SPOP was confirmed by an earlier study that showed that downregulation of SPOP expression in cancers might inhibit its functions as a tumour suppressor gene and might promote cancer development[45]. On the basis of the results of immunohistochemical staining and cell culture experiments in the present study, SPOP may act as a potential tumour suppressor protein in the tumorigenesis of RCC. However, the findings of the survival analysis did not provide supportive evidence showing a correlation of SPOP expression and overall survival, suggesting that high expression of SPOP could not be regarded as a hallmark of RCC and could not yet predict the prognosis of patients. The exact role of the SPOP protein in RCC is controversial and still needs to be confirmed by further research based on a large cohort of samples.

Although there are some important discoveries in the present study, some limitations need to be discussed. First, the concentration gradient of drug experiments was too large to accurately reflect the significant concentration. Second, it is widely accepted that RCC is a heterogeneous tumour with distinct pathological tissue subtypes, including clear cell, papillary, and chromophobe subtypes. The TMA tissues used in this study consisted of a single pathological tissue subtype and could not be used to explore the expression of SPOP in the different subtypes of RCC. More pathological tissue subtypes should be included to analyse the expression of SPOP in RCC tissue in the future, especially fresh frozen tissue from RCC radical nephrectomy.

\section{Conclusions}

In brief, we report that SPOP reduces tumorigenesis features in RCC cell lines and induces cell apoptosis in vitro. In human RCC samples, SPOP is expressed at low levels in the majority of ccRCC samples and at higher levels in most adjacent nontumour samples. All of these findings suggest that SPOP may act as a potential tumour suppressor protein in the tumorigenesis of human RCC. Further studies with a larger patient cohort and analysis of molecular mechanisms are needed to confirm our findings.

\section{Abbreviations}


RCC: renal cell carcinoma; ccRCC: clear cell renal cell carcinoma; SPOP $₫$ Speckle-type POZ protein; TMAs: tissue microarrays; VEGF: Vascular endothelial growth factor;PI3K: Phosphoinositide-3 kinase; AKT: protein kinase B;mTOR:mammalian target of rapamycin; CRL3: cullin3-RING ubiquitin ligase; AR: androgen receptor; SRC: steroid receptor coactivator; PTEN: phosphatase and tensin homolog deleted on

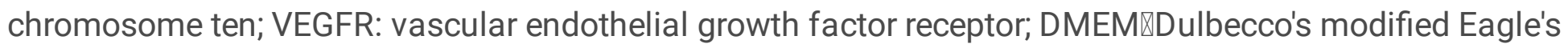
medium. HHIs:Hedgehog inhibitors.

\section{Declarations}

\section{Ethics approval and consent to participate}

The study was approved by the local Ethics Committee (The People's Hospital of Guangxi Zhuang Autonomous Region, No. 2014-010).

\section{Consent for publication}

All authors have given their consent for the publication of this article.

\section{Availability of data and materials}

All data generated or analyzed during this study are included either in this article. The datasets used and/oranalysed during the current study are available from the corresponding author on reasonable request.

\section{Competing interests}

No potential conflicts of interest are disclosed.

\section{Funding}

This study was supported by grants from National Natural Science Foundation of China (No. 81760456 to the principal investigator, Wei Li)

\section{Authors' contributions}

WL: Study concept and design; ZC, ZL and DN: performed the in vitro experiments and assisted with the cell based; ZC, and WL: analysis and interpretation of data; XL, GH and JL: contributed reagents/materials/analysis tools; $Z C$ and WL: supervised the overall experimental design. The author(s) read and approved the final manuscript. 


\section{Acknowledgements}

We thank all members of the Research Center of Medical Sciences (The People's Hospital of Guangxi Zhuang Autonomous Region, 6 Taoyuan Road,Nanning 530021, China) for their support.

\section{Author details}

${ }^{1}$ Department of Urology, The People's Hospital of Guangxi Zhuang Autonomous Region, Nanning, China.

${ }^{2}$ Graduate School, Guangxi Medical University, Nanning, China.

\section{References}

1. Siegel RL, Miller KD, Jemal A: Cancer statistics, 2019. CA Cancer J Clin 2019, 69(1):7-34.

2. Medina-Rico M, Ramos HL, Lobo M, Romo J, Prada JG: Epidemiology of renal cancer in developing countries: Review of the literature. Can Urol Assoc J 2018, 12(3):E154-E162.

3. Dy GW, Gore JL, Forouzanfar MH, Naghavi M, Fitzmaurice C: Global Burden of Urologic Cancers, 1990-2013. European Urology 2017, 71(3):437-446.

4. Warren AY, Harrison D: WHO/ISUP classification, grading and pathological staging of renal cell carcinoma: standards and controversies. World J Urol 2018, 36(12):1913-1926.

5. Rossi SH, Klatte T, Usher-Smith J, Stewart GD: Epidemiology and screening for renal cancer. World J Urol 2018, 36(9):1341-1353.

6. Lalani AA, McGregor BA, Albiges L, Choueiri TK, Motzer R, Powles T, Wood C, Bex A: Systemic Treatment of Metastatic Clear Cell Renal Cell Carcinoma in 2018: Current Paradigms, Use of Immunotherapy, and Future Directions. Eur Urol 2019, 75(1):100-110.

7. Porta C, Gore ME, Rini BI, Escudier B, Hariharan S, Charles LP, Yang L, DeAnnuntis L, Motzer RJ: Longterm Safety of Sunitinib in Metastatic Renal Cell Carcinoma. European Urology 2016, 69(2):345-351.

8. Rajandram R, Bennett NC, Morais C, Johnson DW, Gobe GC: Renal cell carcinoma: resistance to therapy, role of apoptosis, and the prognostic and therapeutic target potential of TRAF proteins. Med Hypotheses 2012, 78(2):330-336.

9. Nagai Y, Kojima T, Muro Y, Hachiya T, Nishizawa Y, Wakabayashi T, Hagiwara M: Identification of a novel nuclear speckle-type protein, SPOP. FEBS Letters 1997, 418(1-2):23-26.

10. Cuneo MJ, Mittag T: The ubiquitin ligase adaptor SPOP in cancer. FEBS J 2019, 286(20):3946-3958.

11. Mani RS: The emerging role of speckle-type POZ protein (SPOP) in cancer development. Drug Discov Today 2014, 19(9):1498-1502.

12. Chen H-Y, Chen R-H: Cullin 3 Ubiquitin Ligases in Cancer Biology: Functions and Therapeutic Implications. Frontiers in Oncology 2016, 6.

13. Song $Y, X u Y$, Pan C, Yan L, Wang ZW, Zhu X: The emerging role of SPOP protein in tumorigenesis and cancer therapy. Mol Cancer 2020, 19(1):2. 
14. Chunyan Zeng YW, Quqin Lu, Jiang Chen, Junyan Zhang, Tao Liu, Nonghua Lv and Shiwen Luo*: SPOP suppresses tumorigenesis by regulating Hedgehog/Gli2 signaling pathway in gastric cancer. Journal of Experimental \& Clinical Cancer Research 2014, 33:75.

15. Blattner M, Liu D, Robinson BD, Huang D, Poliakov A, Gao D, Nataraj S, Deonarine LD, Augello MA, Sailer $\mathrm{V}$ et al: SPOP Mutation Drives Prostate Tumorigenesis In Vivo through Coordinate Regulation of PI3K/mTOR and AR Signaling. Cancer Cell 2017, 31(3):436-451.

16. Kan Z, Jaiswal BS, Stinson J, Janakiraman V, Bhatt D, Stern HM, Yue P, Haverty PM, Bourgon R, Zheng $\mathrm{J}$ et al: Diverse somatic mutation patterns and pathway alterations in human cancers. Nature 2010, 466(7308):869-873.

17. Li G, Ci W, Karmakar S, Chen K, Dhar R, Fan Z, Guo Z, Zhang J, Ke Y, Wang L et al: SPOP promotes tumorigenesis by acting as a key regulatory hub in kidney cancer. Cancer Cell 2014, 25(4):455-468.

18. Zhao W, Zhou J, Deng Z, Gao Y, Cheng Y: SPOP promotes tumor progression via activation of betacatenin/TCF4 complex in clear cell renal cell carcinoma. Int J Oncol 2016, 49(3):1001-1008.

19. Ramakrishnan S, Ku S, Ciamporcero E, Miles KM, Attwood K, Chintala S, Shen L, Ellis L, Sotomayor P, Swetzig $W$ et al: HDAC 1 and 6 modulate cell invasion and migration in clear cell renal cell carcinoma. BMC Cancer 2016, 16(1).

20. Hogner A, Krause H, Jandrig B, Kasim M, Fuller TF, Schostak M, Erbersdobler A, Patzak A, Kilic E: PBRM1 and VHL expression correlate in human clear cell renal cell carcinoma with differential association with patient's overall survival. Urol Oncol 2018, 36(3):94 e91-94 e14.

21. Tochizawa S, Masumori N, Yanai Y, Ohmoto Y, Yabuuchi Y, Tsukamoto T: Antitumor effects of a combination of interferon-alpha and sorafenib on human renal carcinoma cell lines. Biomedical research (Tokyo, Japan) 2008, 29(6):271-278.

22. Liu J, Ghanim M, Xue L, Brown CD, lossifov I, Angeletti C, Hua S, Nègre N, Ludwig M, Stricker T et al: Analysis of Drosophila segmentation network identifies a JNK pathway factor overexpressed in kidney cancer. Science 2009, 323(5918):1218-1222.

23. Theurillat J-PP, Udeshi ND, Errington WJ, Svinkina T, Baca SC, Pop M, Wild PJ, Blattner M, Groner AC, Rubin MA et al: Ubiquitylome analysis identifies dysregulation of effector substrates in SPOP-mutant prostate cancer. Science 2014, 346(6205):85-89.

24. Liu D, Takhar M, Alshalalfa M, Erho N, Shoag J, Jenkins RB, Karnes RJ, Ross AE, Schaeffer EM, Rubin MA et al: Impact of the SPOP Mutant Subtype on the Interpretation of Clinical Parameters in Prostate Cancer. JCO precision oncology 2018, 2018.

25. Guo ZQ, Zheng T, Chen B, Luo C, Ouyang S, Gong S, Li J, Mao LL, Lian F, Yang Y et al: Small-Molecule Targeting of E3 Ligase Adaptor SPOP in Kidney Cancer. Cancer Cel/ 2016, 30(3):474-484.

26. Ji P, Liang S, Li P, Xie C, Li J, Zhang K, Zheng X, Feng M, Li Q, Jiao H et al: Speckle-type POZ protein suppresses hepatocellular carcinoma cell migration and invasion via ubiquitin-dependent proteolysis of SUM01/sentrin specific peptidase 7. Biochemical and Biophysical Research Communications 2018, 502(1):30-42. 
27. Xu J, Wang F, Jiang H, Jiang Y, Chen J, Qin J: Properties and Clinical Relevance of Speckle-Type POZ Protein in Human Colorectal Cancer. Journal of gastrointestinal surgery : official journal of the Society for Surgery of the Alimentary Tract 2015, 19(8):1484-1496.

28. Tan P, Xu Y, Du Y, Wu L, Guo B, Huang S, Zhu J, Li B, Lin F, Yao L: SPOP suppresses pancreatic cancer progression by promoting the degradation of NANOG. Cell death \& disease 2019, 10(11):794.

29. Méjean A, Ravaud A, Thezenas S, Colas S, Beauval JB, Bensalah K, Geoffrois L, Thiery-Vuillemin A, Cormier L, Lang H et al: Sunitinib Alone or after Nephrectomy in Metastatic Renal-Cell Carcinoma. The New England journal of medicine 2018, 379(5):417-427.

30. Guba M, von Breitenbuch P, Steinbauer M, Koehl G, Flegel S, Hornung M, Bruns CJ, Zuelke C, Farkas $S$, Anthuber $M$ et al: Rapamycin inhibits primary and metastatic tumor growth by antiangiogenesis: involvement of vascular endothelial growth factor. Nature medicine 2002, 8(2):128-135.

31. Le Tourneau C, Faivre S, Serova M, Raymond E: mTORC1 inhibitors: is temsirolimus in renal cancer telling us how they really work? Br J Cancer 2008, 99(8):1197-1203.

32. Sharma S, Guru SK, Manda S, Kumar A, Mintoo MJ, Prasad VD, Sharma PR, Mondhe DM, Bharate SB, Bhushan S: A marine sponge alkaloid derivative 4-chloro fascaplysin inhibits tumor growth and VEGF mediated angiogenesis by disrupting PI3K/Akt/mTOR signaling cascade. Chem Biol Interact 2017, 275:47-60.

33. Revathidevi S, Munirajan AK: Akt in cancer: Mediator and more. Semin Cancer Biol 2019, 59:80-91.

34. Vanhaesebroeck B, Stephens L, Hawkins P: PI3K signalling: the path to discovery and understanding. Nat Rev Mol Cell Biol 2012, 13(3):195-203.

35. Pantuck AJ, Seligson DB, Klatte T, Yu H, Leppert JT, Moore L, O'Toole T, Gibbons J, Belldegrun AS, Figlin RA: Prognostic relevance of the mTOR pathway in renal cell carcinoma: implications for molecular patient selection for targeted therapy. Cancer 2007, 109(11):2257-2267.

36. Pal SK, Quinn DI: Differentiating mTOR inhibitors in renal cell carcinoma. Cancer Treat Rev 2013, 39(7):709-719.

37. Zhang S, Xiao J, Chai Y, Hong Z, Liu Z, Yuan R, Luo Z, Zhou X, Lucero-Prisno DE, III, Huang K: Speckle-Type POZ Protein Down-Regulates Matrix Metalloproteinase 2 Expression via Sp1/PI3K/Akt Signaling Pathway in Colorectal Cancer. Dig Dis Sci 2018, 63(2):395-402.

38. Chen L, Pei H, Lu SJ, Liu ZJ, Yan L, Zhao XM, Hu B, Lu HG: SPOP suppresses osteosarcoma invasion via PI3K/AKT/NF-KB signaling pathway. European review for medical and pharmacological sciences 2018, 22(3):609-615.

39. Skoda AM, Simovic D, Karin V, Kardum V, Vranic S, Serman L: The role of the Hedgehog signaling pathway in cancer: A comprehensive review. Bosn J Basic Med Sci 2018, 18(1):8-20.

40. Dormoy V, Danilin S, Lindner V, Thomas L, Rothhut S, Coquard C, Helwig JJ, Jacqmin D, Lang H, Massfelder $\mathrm{T}$ : The sonic hedgehog signaling pathway is reactivated in human renal cell carcinoma and plays orchestral role in tumor growth. Mol Cancer 2009, 8:123.

41. Jager W, Thomas C, Fazli L, Hurtado-Coll A, Li E, Janssen C, Gust KM, So Al, Hainz M, Schmidtmann I et al: $\mathrm{DHH}$ is an independent prognosticator of oncologic outcome of clear cell renal cell carcinoma. 
J Urol 2014, 192(6):1842-1848.

42. Zhou J, Zhu G, Huang J, Li L, Du Y, Gao Y, Wu D, Wang X, Hsieh JT, He D et al: Non-canonical GLI1/2 activation by PI3K/AKT signaling in renal cell carcinoma: A novel potential therapeutic target. Cancer Lett 2016, 370(2):313-323.

43. Cortes JE, Gutzmer R, Kieran MW, Solomon JA: Hedgehog signaling inhibitors in solid and hematological cancers. Cancer Treat Rev 2019, 76:41-50.

44. Li JJ, Zhang JF, Yao SM, Huang H, Zhang S, Zhao M, Huang JA: Decreased expression of speckletype POZ protein for the prediction of poor prognosis in patients with non-small cell lung cancer. Oncol Lett 2017, 14(3):2743-2748.

45. Kim MS, Je EM, Oh JE, Yoo NJ, Lee SH: Mutational and expressional analyses of SPOP, a candidate tumor suppressor gene, in prostate, gastric and colorectal cancers. APMIS 2013, 121(7):626-633.

\section{Figures}

Figure 1

A

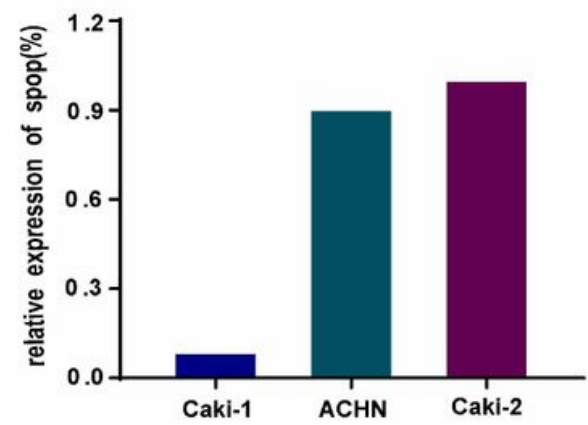

C

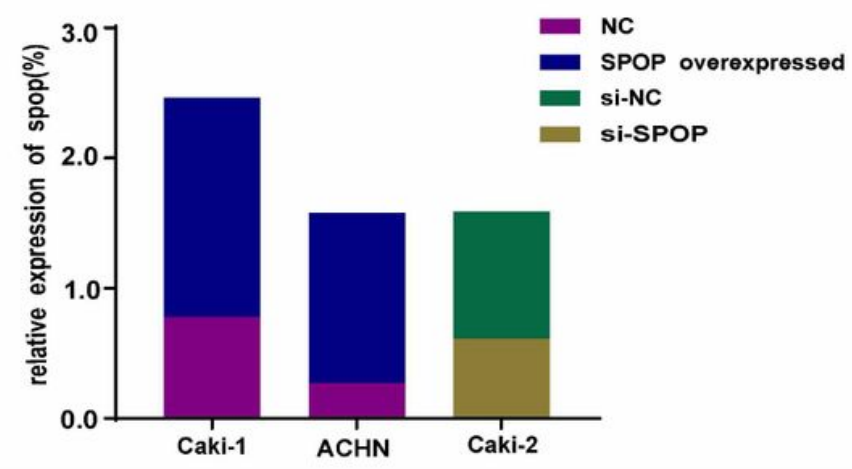

$\mathrm{B}$

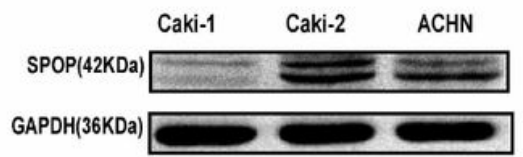

D

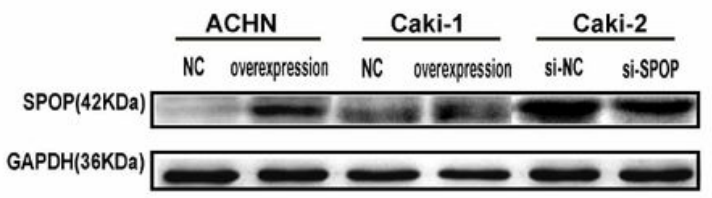

Figure 1 
Expression of SPOP in ACHN, Caki-1 and Caki-2 cells. (A and B) Relative expression of SPOP in RCC cell lines was measured by western blot analysis. SPOP expression in Caki-2 cells was highest in the above RCC cell lines. (C and D) SPOP was knocked down in Caki-2 cells and upregulated in ACHN and Caki-1 cells through transfection with a lentiviral vector for further experiments. The levels of SPOP in RCC cell lines were determined by western blot and QPCR analyses. ACHN and Caki-1 cells were divided into the NC group and SPOP-overexpressing group. Caki-2 cells were divided into the si-NC and si-SPOP groups.

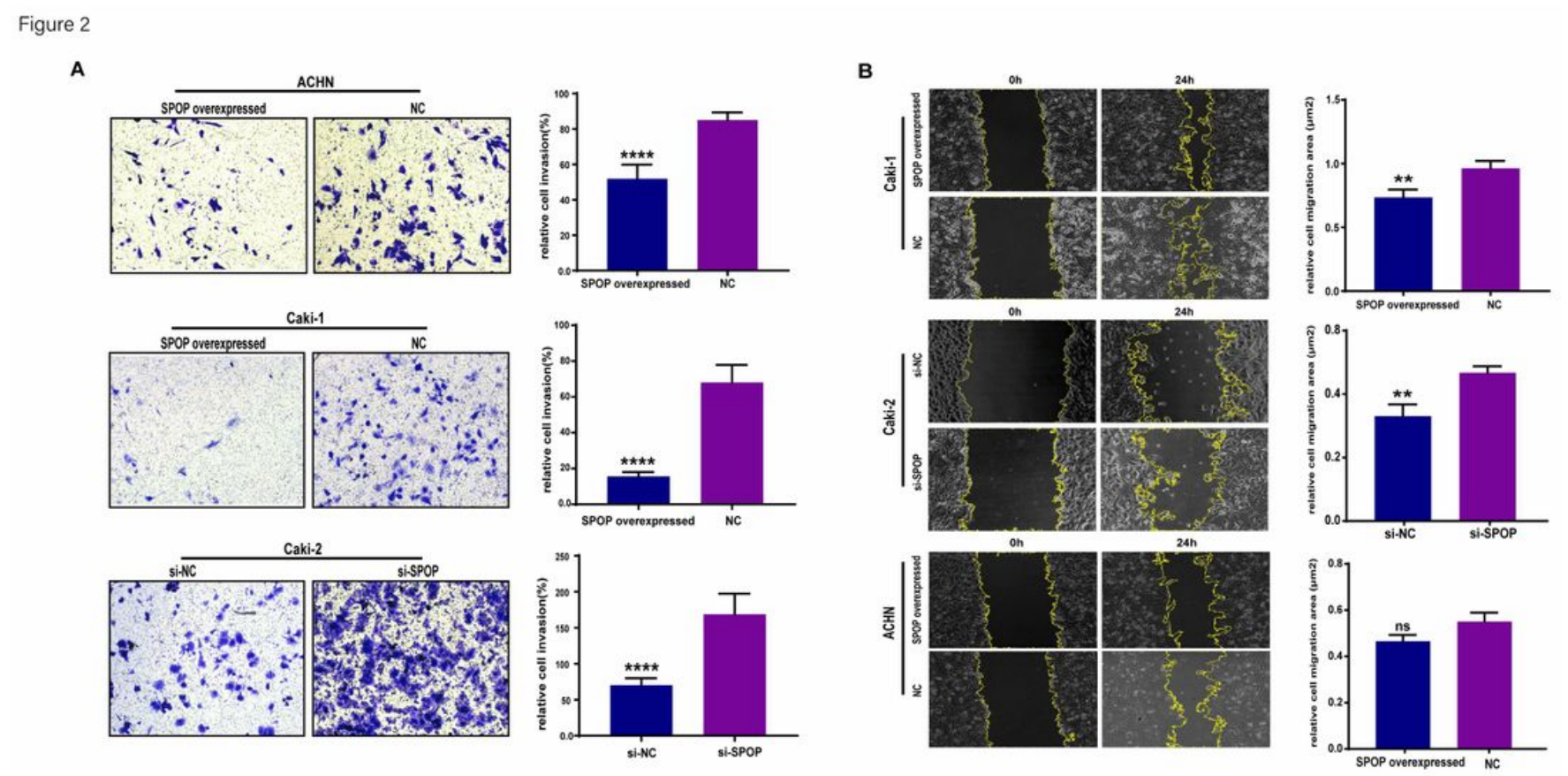

\section{Figure 2}

The effect of SPOP expression on the invasion and migration of RCC cells. (A) Five high-power fields under a microscope were randomly selected to count the number of invading cells after incubation in Transwells for $48 \mathrm{~h}$. Cell invasion was significantly decreased in the SPOP high expression group compared with the SPOP low expression group ( $* \star \star \star, P<0.0001)$. (B) Cell migration was determined by the wound-healing assay, and the scratch migration area was calculated using Image J software after $0 \mathrm{~h}$ and $24 \mathrm{~h}$. Overexpression of SPOP significantly inhibited the invasion ability of Caki-1 and Caki-2 cells (**, $P<0.01)$, and a trend towards a statistically significant difference was observed in ACHN cells. $(P=0.0649$, ns) 
Figure 3

A

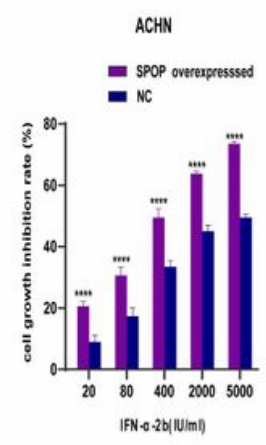

B

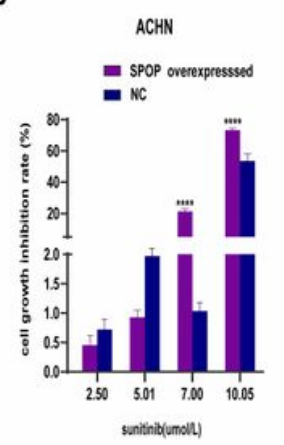

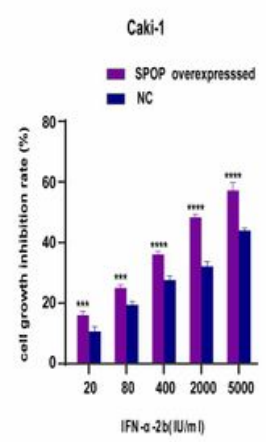
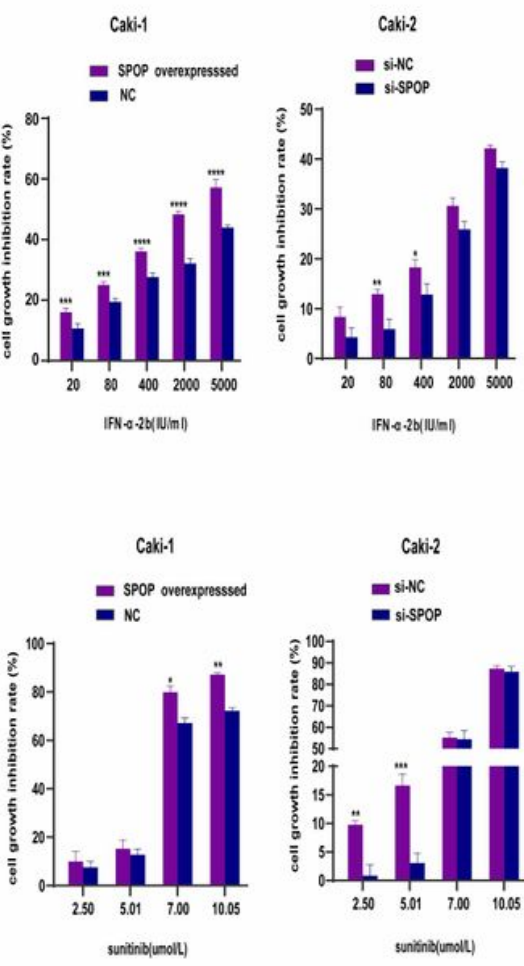

C

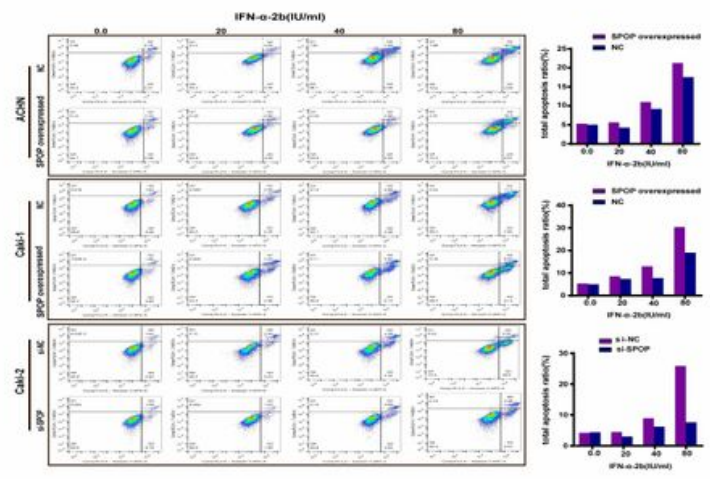

D

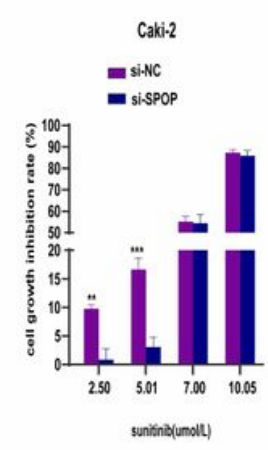

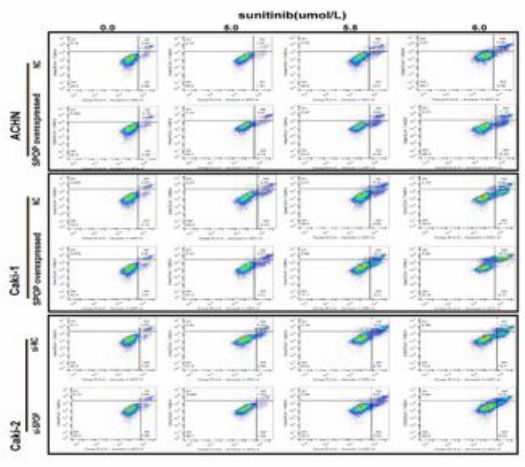

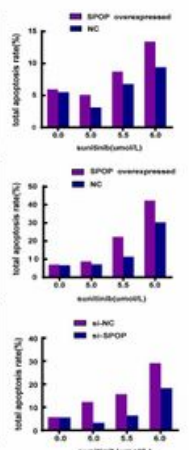

Figure 3

The effects of upregulation or knockdown of SPOP under treatment with sunitinib or IFN- $a 2 b$ on RCC cell proliferation and apoptosis. (A and B) The cells were incubated with the indicated drug concentrations for $48 \mathrm{~h}$ and subjected to ELISA to analyse OD450 and calculate the proliferation inhibitory rate of the cells. Sunitinib or IFN- $a 2 b$ at several concentrations significantly increased the proliferation rate of RCC cells overexpressing SPOP compared to that of RCC cells with low expression of SPOP $\left({ }^{*}, P<0.05, * \star, P<0.01\right.$. $\star \star \star, P<0.001 . * \star \star \star, P<0.0001)$. ( $C$ and $D)$ RCC cells were treated with different concentrations of sunitinib or IFN-a2b, collected and stained with Annexin V-APC and 7-AAD. The total apoptosis rate consisted of early apoptosis (Q3) and late apoptosis (Q2) and was quantified by using flow cytometry. The total apoptosis rate of RCC cells overexpressing SPOP increased with the increasing drug concentrations, and the SPOP protein may improve the susceptibility of RCC cells to drug treatments. 
Figure 4
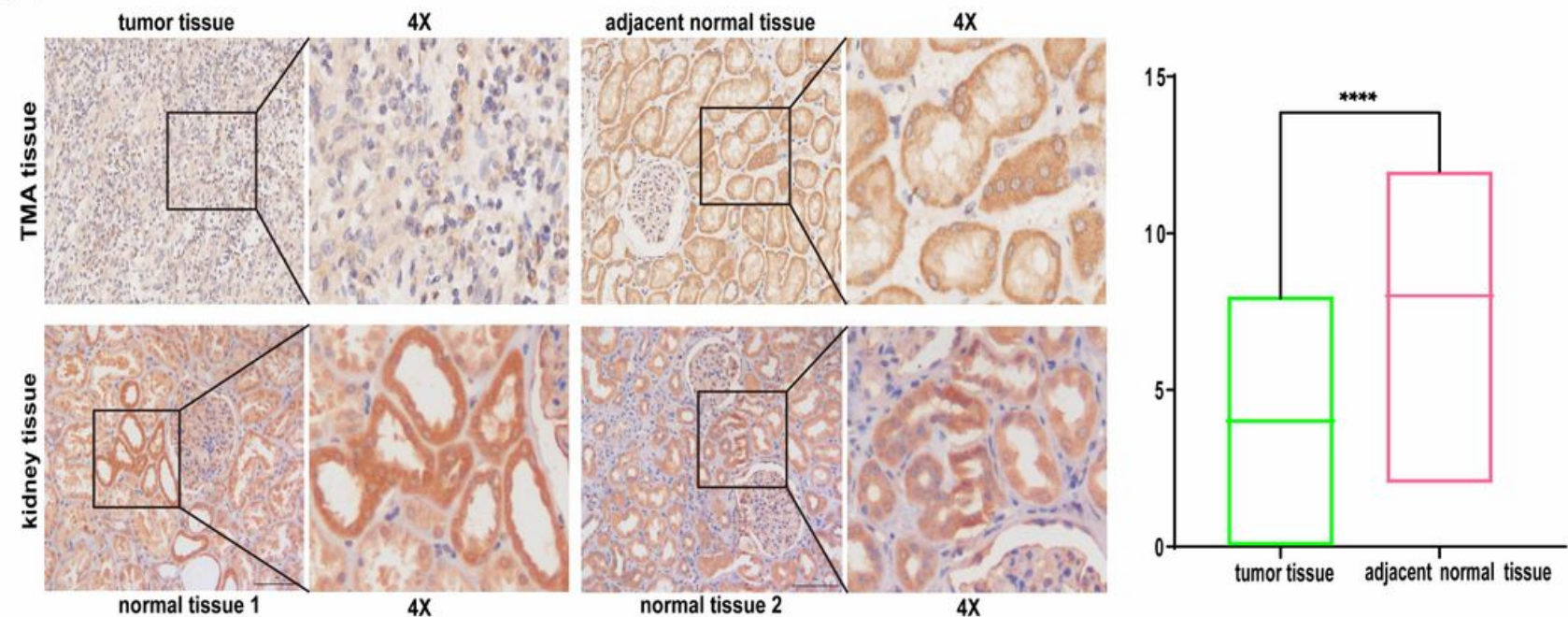

\section{Figure 4}

Immunohistochemical staining of SPOP in the cytoplasm of RCC tissues and adjacent normal tissue. SPOP expression in the TMA was assessed by Aperio image software, and the image was captured at $4 \mathrm{X}$ magnification. In the TMA, SPOP was significantly expressed at low levels in the cytoplasm of ccRCC tissues and at high levels in adjacent normal tissue $(\star \star \star \star, ~ P<0.0001)$. Similar results were observed in normal kidney tissue overexpressing the SPOP protein under a $4 \mathrm{X}$ field microscope. 
Figure 5
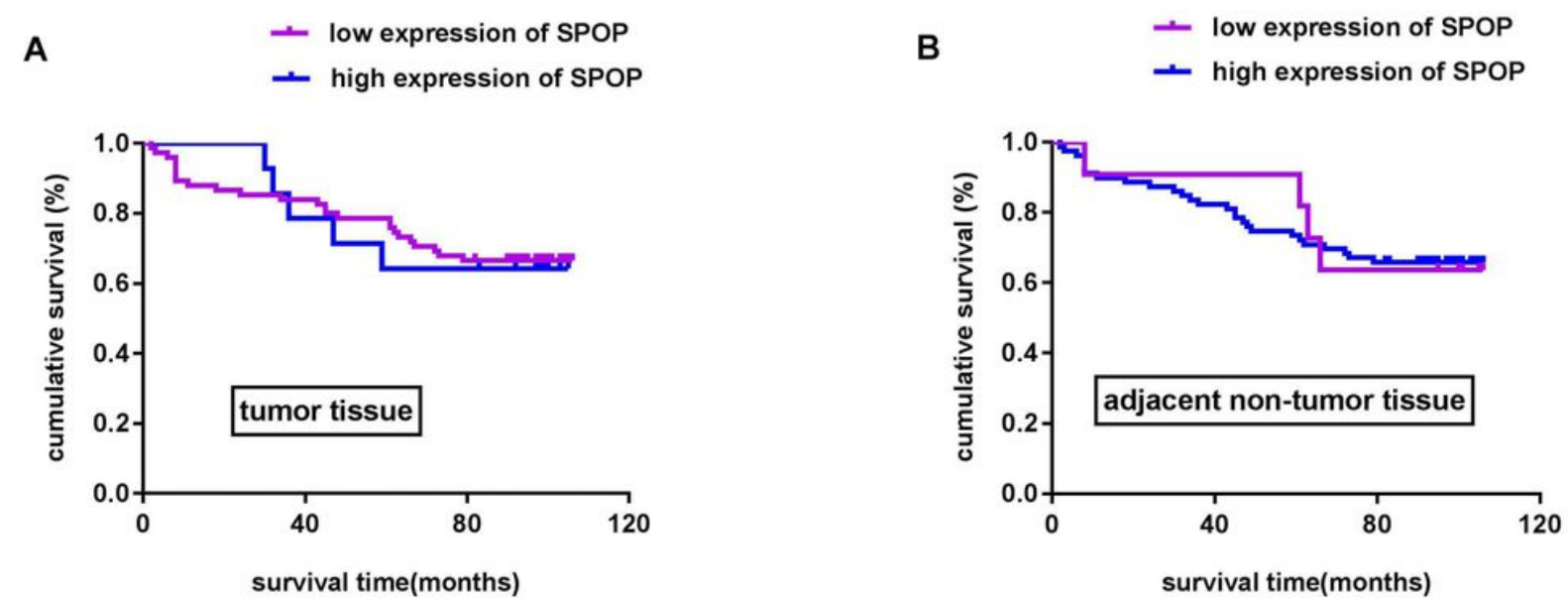

\section{Figure 5}

Kaplan-Meier survival analysis. (A) No statistically significant survival difference was noted between patients with high or low expression of SPOP in tumour tissue $(P>0.05)$. (B) There was no statistically significant survival difference between patients with different expression levels of SPOP in adjacent nontumour tissue $(P>0.05)$. 